\title{
Semiclassical description of electron dynamics in extended systems under intense laser fields
}

\author{
Mizuki Tani $\odot,{ }^{1,2, *}$ Tomohito Otobe $\odot,{ }^{2, \dagger}$ Yasushi Shinohara $\odot^{1,3}$ and Kenichi L. Ishikawa $\odot^{1,3,4, \dagger}$ \\ ${ }^{1}$ Department of Nuclear Engineering and Management, Graduate School of Engineering, The University of Tokyo, \\ 7-3-1 Hongo, Bunkyo-ku, Tokyo 113-8656, Japan \\ ${ }^{2}$ Kansai Photon Science Institute, National Institutes for Quantum and Radiological Science and Technology (QST), Kyoto 619-0215, Japan \\ ${ }^{3}$ Photon Science Center, Graduate School of Engineering, The University of Tokyo, 7-3-1 Hongo, Bunkyo-ku, Tokyo 113-8656, Japan \\ ${ }^{4}$ Research Institute for Photon Science and Laser Technology, The University of Tokyo, 7-3-1 Hongo, Bunkyo-ku, Tokyo 113-0033, Japan
}

(Received 17 May 2021; revised 27 July 2021; accepted 2 August 2021; published 30 August 2021)

\begin{abstract}
We propose a semiclassical approach based on the Vlasov equation to describe the time-dependent electronic dynamics in a bulk simple metal under an ultrashort intense laser pulse. We include in the effective potential not only the ionic Coulomb potential and mean-field electronic Coulomb potential from the one-body electron distribution but also the exchange-correlation potential within the local-density approximation (LDA). The initial ground state is obtained by the Thomas-Fermi model. To numerically solve the Vlasov equation, we extend the pseudoparticle method, previously used for nuclei and atomic clusters, to solids, taking the periodic boundary condition into account. We apply the present implementation to a bulk aluminum (fcc) conventional unit cell irradiated with a short laser pulse. The optical conductivity, refractive index, extinction coefficient, and reflectivity as well as energy absorption calculated with the Vlasov-LDA method are in excellent agreement with the results by the time-dependent density-functional theory and experimental references.
\end{abstract}

DOI: 10.1103/PhysRevB.104.075157

\section{INTRODUCTION}

The interaction of ultrashort (fs-ps) intense laser pulses with solids is relevant to a wide area of research ranging from high-order harmonic generation [1-5] to material machining [6-14]. The process of ultrafast laser micromachining, which can suppress heat-affected zones, starts from the energy transfer from the laser to the material by electron excitation, followed by that from the hot electrons to the lattice. As a result, the material undergoes phase and/or structural transition [15], leaving a change of the optical constants or a defect behind [16], which eventually leads to ablation, drilling, or structuring [17-32].

The comprehensive modeling of laser material machining is highly complex, multiscale in both time and space, multiphase (solid, fluid, plasma, cluster, etc.), and possibly accompanied by chemical reactions. Plasma or continuum models [6,8,18,33-35], for example, have been employed to describe and simulate such processes, advancing understanding. However, they have difficulties in examining initial transient dynamics before the local thermodynamic equilibrium is reached.

*mzktani@atto.t.u-tokyo.ac.jp

${ }^{\dagger}$ Corresponding authors: otobe.tomohito@qst.go.jp; ishiken@n.t.u-tokyo.ac.jp

Published by the American Physical Society under the terms of the Creative Commons Attribution 4.0 International license. Further distribution of this work must maintain attribution to the author(s) and the published article's title, journal citation, and DOI.
It has now become possible to describe the attosecondfemtosecond electron dynamics under intense laser fields with the time-dependent density-functional theory (TDDFT) [36-39] or time-dependent density-matrix methods [40-42]. TDDFT is an ab initio method that offers a good compromise between accuracy and computational feasibility. Its computational cost is, however, still very high, especially, if one wants to perform long-timescale simulation, coupling it with molecular dynamics and electromagnetic-field analysis.

In TDDFT, each electron orbital satisfies the timedependent Kohn-Sham (TDKS) equation [see Eq. (1) below]. The leading order of a semiclassical $\hbar$ expansion of the TDKS equation reduces to the Vlasov equation, which describes the temporal evolution of the electron distribution function in phase space. Thus, Vlasov-based approaches are expected to be a cost-effective alternative to TDDFT, in particular, for metals. Such approaches have previously been applied to ionization and explosion dynamics of molecules $[43,44]$ and metal clusters [45-50]. The Vlasov equation is numerically solved with so-called pseudoparticle methods in these studies, which represent the electron cloud as an assembly of classical test particles whose motion is governed by Newton's equations of motion. There are several reports of application to $\mathrm{Na}$ clusters, well agreeing with TDDFT results [48-52].

In this paper, we extend the pseudoparticle method based on the Vlasov equation to the description of electron dynamics in extended systems under intense laser fields. The effective potential acting on the electrons contains not only the ionic potential, interelectronic Hartree potential, and interaction with laser but also the exchange-correlation potential within the local-density approximation (LDA), and incorporates the periodic boundary condition. We apply the present method to 
bulk aluminum. The calculated optical conductivity, refractive index, extinction coefficient, and reflectivity as well as energy absorption are in excellent agreement with TDDFT calculations and experimental references.

The present paper is organized as follows. Section II describes our simulation methods. We review the Vlasov equation and describe our numerical implementations with the periodic boundary condition. In Sec. III we describe numerical application to bulk aluminum and compare the results with TDDFT and measurement values. The conclusions are given in Sec. IV.

\section{METHODS}

\section{A. Vlasov equation}

Among the methods for treating quantum many-body dynamics, TDDFT provides a feasible computational framework for treating electronic systems' optical response or charged particles' collision phenomena [53]. The time propagation of a $N_{e}$-electron system comes down to solving a set of equations for the Kohn-Sham orbitals $\left\{\phi_{i}(\mathbf{r}, t)\right\}$ that evolve in a selfconsistent mean field [54],

$$
i \hbar \frac{\partial}{\partial t} \phi_{i}(\mathbf{r}, t)=h_{\mathrm{KS}}\left[n_{e}(\mathbf{r}, t)\right] \phi_{i}(\mathbf{r}, t),
$$

where

$$
h_{\mathrm{KS}}\left[n_{e}(\mathbf{r}, t)\right]=-\frac{\hbar^{2}}{2 m} \nabla^{2}+V_{\mathrm{eff}}\left[n_{e}(\mathbf{r}, t)\right]
$$

denotes the Kohn-Sham Hamiltonian, $m$ the electron mass, $V_{\text {eff }}$ the effective potential (see below), and the time-dependent electron density $n_{e}(\mathbf{r}, t)$ is defined as

$$
n_{e}(\mathbf{r}, t)=\sum_{i=1}^{N_{e}}\left|\phi_{i}(\mathbf{r}, t)\right|^{2} .
$$

Analogously, the density operator $\hat{\rho}(t)$ is defined as

$$
\left\langle\mathbf{r}|\hat{\rho}(t)| \mathbf{r}^{\prime}\right\rangle=\sum_{i=1}^{N_{e}} \phi_{i}^{*}(\mathbf{r}, t) \phi_{i}\left(\mathbf{r}^{\prime}, t\right),
$$

whose evolution is governed by the von Neumann equation (vNE),

$$
\frac{\partial}{\partial t} \hat{\rho}(t)=-\frac{i}{\hbar}\left[\hat{h}_{\mathrm{KS}}(t), \hat{\rho}(t)\right] .
$$

Performing the Wigner transformation [55] and taking the limit $\hbar \rightarrow 0$, the density operator $\hat{\rho}(t)$ is mapped onto a real function $f(\mathbf{r}, \mathbf{p}, t)$, which obeys the Vlasov equation,

$$
\begin{aligned}
\frac{\partial}{\partial t} f(\mathbf{r}, \mathbf{p}, t)= & -\frac{\mathbf{p}}{m} \cdot \nabla_{\mathbf{r}} f(\mathbf{r}, \mathbf{p}, t) \\
& +\nabla_{\mathbf{r}} V_{\mathrm{eff}}\left[n_{e}(\mathbf{r}, t)\right] \cdot \nabla_{\mathbf{p}} f(\mathbf{r}, \mathbf{p}, t),
\end{aligned}
$$

which is a classical alternative to the vNE, Eq. (5), where $\mathbf{p}$ is the electron canonical momentum. Here, $f(\mathbf{r}, \mathbf{p}, t)$ is interpreted as the electron distribution in phase space. Since the Vlasov equation cannot describe discrete levels, the present approach is justified for metals under situations where interband transitions play a relatively minor role.
The effective potential $V_{\text {eff }}$ is a functional of the electron density distribution $n_{e}(\mathbf{r}, t)$ and decomposed into

$$
V_{\text {eff }}\left[n_{e}(\mathbf{r}, t)\right]=V_{\text {Coulomb }}\left[n_{e}(\mathbf{r}, t)\right]+V_{\mathrm{xc}}\left[n_{e}(\mathbf{r}, t)\right]+V_{\text {ext }}(\mathbf{r}, t),
$$

with the exchange-correlation potential $V_{\mathrm{xc}}$, external field potential $V_{\text {ext }}$, and

$$
V_{\text {Coulomb }}\left[n_{e}(\mathbf{r}, t)\right]=\sum_{i} V_{\mathrm{ps}}\left(\mathbf{r}-\mathbf{r}_{i}\right)+V_{\mathrm{H}}\left[n_{e}(\mathbf{r}, t)\right],
$$

where $i, V_{\mathrm{ps}}$, and $V_{\mathrm{H}}$ denote the label of ions and the spherically symmetric ionic pseudopotential and the electronelectron Hartree potential, respectively.

Several previous works for $\mathrm{Na}$ clusters have used their original pseudopotentials $[45,46]$, adjusted so that the simulation results reproduce the static and dynamical properties of the system. In this work, instead, we employ the modified Heine-Abarenkov type local pseudopotential for $V_{\mathrm{ps}}$,

$$
V_{\mathrm{ps}}(r)=-\frac{z}{R} e\left\{\frac{1}{r}\left[1-(1+\beta r) e^{-\alpha r}-A e^{-r}\right]\right\}(r=|\mathbf{r}|),
$$

where $z$ is the number of the valence electrons, and $A, R, \alpha$, and $\beta$ are material-dependent parameters determined by $a b$ initio density-functional formalism in Ref. [56], thus, independent from Vlasov simulations. Their values for the bulk aluminum crystal are $A=3.574$ a.u., $\alpha=3.635$ a.u., $\beta=$ 0.8343 a.u., $R=0.334$ a.u., and $z=3$. $V_{\mathrm{H}}$ is evaluated by solving the Poisson equation,

$$
\Delta V_{\mathrm{H}}\left[n_{e}(\mathbf{r}, t)\right]=-4 \pi e n_{e}(\mathbf{r}, t) .
$$

Here, let us introduce a real-space simulation box $\Omega$, on which the periodic boundary condition is imposed, and translation vectors $\mathbf{G} . \Omega$ is defined as

$$
\Omega=\left\{\mathbf{r}=\sum_{j=x, y, z} a_{j} \mathbf{e}_{j} \mid 0 \leqslant a_{j}<1\right\},
$$

where $\left\{\mathbf{e}_{j}\right\}$ are the lattice vectors along the $j$ axis $(j=x, y, z)$, whose lengths are denoted by $L_{j}=\left|\mathbf{e}_{j}\right|$. Integrals with respect to $\mathbf{r}$ are taken over $\Omega$ in what follows. The translation vectors are given by

$$
\mathbf{G}=\sum_{j=x, y, z} M_{j} \mathbf{e}_{j}\left(M_{j}=0, \pm 1, \pm 2, \ldots\right) .
$$

Taking the periodic boundary condition into account, the Coulomb terms $V_{\mathrm{ps}}$ and $V_{\mathrm{H}}$ are represented as a Fourier series expansion. The pseudopotential term is rewritten as

$$
\sum_{i}^{\infty} V_{\mathrm{ps}}\left(\mathbf{r}-\mathbf{r}_{i}\right)=\sum_{\mathbf{G}, i=1}^{N_{\mathrm{ion}}} V_{\mathrm{ps}}\left(\mathbf{r}-\mathbf{r}_{i}-\mathbf{G}\right),
$$

where $N_{\text {ion }}$ denotes the number of ions in $\Omega$ and

$$
\begin{aligned}
& \sum_{\mathbf{G}} V_{\mathrm{ps}}\left(\mathbf{r}-\mathbf{r}_{i}-\mathbf{G}\right) \\
& =\mathcal{F}^{-1}\left[\sum_{i} e^{-\mathbf{Q} \cdot \mathbf{r}_{i}}\left\{V_{\mathrm{ps}}(Q)+\frac{4 \pi}{Q} z\right\}\right]
\end{aligned}
$$


with $\mathbf{Q}$ being the coordinates in the Fourier domain $(Q=|\mathbf{Q}|)$, $\mathcal{F}[\cdot]$ the Fourier series expansion within $\Omega$, and

$$
\begin{aligned}
V_{\mathrm{ps}}(Q)= & 4 \pi z e R^{2}\left[-\frac{1}{(Q R)^{2}}+\frac{1}{(Q R)^{2}+\alpha^{2}}\right. \\
& \left.+\frac{2 \alpha \beta}{\left\{(Q R)^{2}+\alpha^{2}\right\}^{2}}+\frac{2 A}{\left\{(Q R)^{2}+1\right\}^{2}}\right] .
\end{aligned}
$$

One obtains the solution of the Poisson equation [Eq. (10)] for the electron density $n_{e}$ given within $\Omega$ as

$$
V_{\mathrm{H}}\left[n_{e}(\mathbf{r}, t)\right]=\mathcal{F}^{-1}\left[\mathcal{F}\left[n_{e}(\mathbf{r}, t)\right] \frac{4 \pi e}{Q^{2}}\right] .
$$

For the exchange-correlation potential $V_{\mathrm{xc}}$, one employs the LDA by Perdew and Zunger [57]. The laser-electron interaction is described in the length gauge,

$$
V_{\mathrm{ext}}(\mathbf{r}, t)=-e \mathbf{E}(t) \cdot \mathbf{r},
$$

within the dipole approximation, where $\mathbf{E}$ denotes the laser electric field vector. In this case, $\mathbf{p}$ becomes the kinetic momentum, and, thus, the electronic current density $\mathbf{J}(t)$ averaged over $\Omega$ is given by

$$
\mathbf{J}(t)=\frac{1}{|\Omega|} \iint_{\Omega}\left(-e \frac{\mathbf{p}}{m}\right) f(\mathbf{r}, \mathbf{p}, t) d \mathbf{r} d \mathbf{p} .
$$

\section{B. Numerical implementations}

\section{Pseudoparticle method}

The direct propagation of the distribution function would require the treatment of six-dimensional time-dependent function on grids [58]. To avoid such a massive computation, one introduces the pseudoparticle method [45-47,59], where the distribution function $f(\mathbf{r}, \mathbf{p}, t)$ is expressed by a set of pseudoparticles with mass $m$ as

$$
f(\mathbf{r}, \mathbf{p}, t)=\frac{1}{N_{s}} \sum_{i=1}^{N_{\mathrm{pp}}} g_{r}\left[\mathbf{r}-\mathbf{r}_{i}(t)\right] g_{p}\left[\mathbf{p}-\mathbf{p}_{i}(t)\right] .
$$

Here $\mathbf{r}_{i}, \mathbf{p}_{i}$ are the position and canonical momentum of each pseudoparticle labeled by $i$. The total number of pseudoparticles $N_{\mathrm{pp}}$ is given by $N_{\mathrm{pp}}=N_{s} N_{e}$, where $N_{s}$ and $N_{e}$ are the number of pseudoparticles per electron and the total number of the electrons contained in $\Omega$, respectively. Statistical error is reduced by increasing $N_{s}$. $N_{s}$ is set to 10000 in this study. $g_{r}(\mathbf{r})$ and $g_{p}(\mathbf{p})$ denote smoothing kernel functions for the position and momentum, respectively, of Gaussian forms,

$$
\begin{gathered}
g_{r}(\mathbf{r})=\sum_{\{\mathbf{G}\}} \frac{1}{\pi^{3 / 2} d_{r}^{3}} \exp \left(-|\mathbf{r}+\mathbf{G}|^{2} / d_{r}^{2}\right), \\
g_{p}(\mathbf{p})=\frac{1}{\pi^{3 / 2} d_{p}^{3}} \exp \left(-|\mathbf{p}|^{2} / d_{p}^{2}\right),
\end{gathered}
$$

where $d_{r}$ and $d_{p}$ are smoothing widths. Only the nearest neighbor cells are included in summation over $\mathbf{G}$ in Eq. (20). The kernel functions are normalized as

$$
\begin{aligned}
& \int_{\Omega} g_{r}(\mathbf{r}) d \mathbf{r}=1, \\
& \int g_{p}(\mathbf{p}) d \mathbf{p}=1,
\end{aligned}
$$

so that

$$
\iint_{\Omega} f(\mathbf{r}, \mathbf{p}, t) d \mathbf{r} d \mathbf{p}=N_{e}
$$

The scattering cross section of the electron and the effective potential is adjusted through $d_{r}$; the smaller $d_{r}$, the larger the cross section. Here we use $d_{r}=1.1$ a.u. so that the linear response, to be discussed below in Fig. 3, is in overall agreement with that obtained by TDDFT in the spectral range between 2 and $25 \mathrm{eV}$. In the present collisionless case, $d_{p}$ is not used explicitly.

The field quantities such as $V_{\text {eff }}$ and $n_{e}$ are evaluated on three-dimensional grids discretized into $N_{j}(j=x, y, z)$ intervals on the $j$ axis with a spatial step $\Delta j=L_{j} / N_{j}$. In our calculation we set $\Delta x=\Delta y=\Delta z=0.95$ a.u. Here, $d_{r} / \Delta x \simeq 1.15$ is a good parametrization leading to stable simulation [46]. It should be noted that $d_{r}$ is the only adjustable parameter in our formalism. The electron density on a grid point $\mathbf{r}$ is calculated as

$$
n_{e}(\mathbf{r}, t)=\int d \mathbf{p} f(\mathbf{r}, \mathbf{p}, t)=\frac{1}{N_{s}} \sum_{i=1}^{N_{\mathrm{pp}}} g_{r}\left[\mathbf{r}_{i}(t)-\mathbf{r}\right] .
$$

The current density $\mathbf{J}(t)$ [Eq. (18)] is evaluated as

$$
\mathbf{J}(t)=-\frac{1}{|\Omega|} \frac{e}{N_{s}} \sum_{i=1}^{N_{\mathrm{pp}}} \frac{\mathbf{p}_{i}(t)}{m} .
$$

The Hamiltonian in pseudoparticle representation is written as

$$
H_{\mathrm{pp}}=\frac{1}{N_{s}} \sum_{i}^{N_{\mathrm{pp}}}\left[\frac{\mathbf{p}_{i}^{2}(t)}{2 m}+\int_{\Omega} V_{\mathrm{eff}}(\mathbf{r}, t) g_{r}\left(\mathbf{r}_{i}-\mathbf{r}\right) d \mathbf{r}\right] .
$$

The motion of each pseudoparticle is governed by the Newton equations under the effective potential $V_{\text {eff }}$ with the periodic boundary condition as

$$
\dot{\mathbf{r}}_{i}=\frac{\mathbf{p}_{i}}{m}, \quad \dot{\mathbf{p}}_{i}=-\int_{\Omega} V_{\text {eff }}(\mathbf{r}) \nabla_{\mathbf{r}_{i}} g_{r}\left(\mathbf{r}_{i}-\mathbf{r}\right) d \mathbf{r} .
$$

As long as pseudoparticle canonical variables $\mathbf{r}_{i}, \mathbf{p}_{i}$ obey the Newton equation [Eq. (28)], one-body distribution [Eq. (19)] satisfies the Vlasov equation [Eq. (6)]. The force term is given as the gradient of the $N_{\mathrm{pp}}$-body Hamiltonian $H_{\mathrm{pp}}$. One numerically integrates it as

$$
\begin{aligned}
& \int V_{\mathrm{eff}}(\mathbf{r}) \nabla_{\mathbf{r}} g_{r}\left(\mathbf{r}_{i}-\mathbf{r}\right) d \mathbf{r} \\
& \quad \simeq \sum_{\mathbf{r} \in \Omega} V_{\mathrm{eff}}(\mathbf{r}) \nabla_{\mathbf{r}_{i}} g_{r}\left(\mathbf{r}_{i}-\mathbf{r}\right) \Delta x \Delta y \Delta z,
\end{aligned}
$$

using the analytical form of $\nabla_{\mathbf{r}_{i}} g_{r}\left(\mathbf{r}_{i}-\mathbf{r}\right)$,

$$
\begin{aligned}
& \nabla_{\mathbf{r}_{i}} g_{r}\left(\mathbf{r}_{i}-\mathbf{r}\right) \\
& \quad=\sum_{\{\mathbf{G}\}} \frac{-2\left(\mathbf{r}_{i}-\mathbf{r}+\mathbf{G}\right)}{\pi^{3 / 2} d_{r}^{5}} \exp \left(-\left|\mathbf{r}_{i}-\mathbf{r}+\mathbf{G}\right|^{2} / d_{r}^{2}\right) .
\end{aligned}
$$

The integration of Eq. (28) is performed by the Verlet method [60] with time step $\Delta t=0.02$ a.u. Particles exiting $\Omega$ are to reenter $\Omega$ from the other side. 
1: procedure Grolnd STATE PREPARATION

2: (-Initialization-)

3: $\quad$ initial guess of $\mu$ and $n_{c}^{\text {in }}$

$4:$

$5:$

$6:$

\section{6: end procedure}

FIG. 1. Algorithm for the ground state preparation.

\section{Ground state}

The initial state is the stationary solution of the Vlasov equation described by the Thomas-Fermi model. The total energy functional,

$$
\begin{aligned}
E_{\mathrm{all}}\left[n_{e}(\mathbf{r})\right]= & \int_{\Omega}\left[\frac{3}{10} \frac{\hbar^{2}\left(3 \pi^{2}\right)^{2 / 3}}{m} n_{\mathrm{e}}^{5 / 3}(\mathbf{r})+\frac{1}{2} V_{\mathrm{H}}(\mathbf{r}) n_{e}(\mathbf{r})\right. \\
& \left.+\sum_{\mathbf{G}, i=1}^{N_{\mathrm{ion}}} V_{\mathrm{ps}}\left(\mathbf{r}-\mathbf{r}_{i}-\mathbf{G}\right) n_{e}(\mathbf{r})+E_{\mathrm{xc}}\left[n_{e}(\mathbf{r})\right]\right] \mathbf{r},
\end{aligned}
$$

is variationally minimized with respect to $n_{e}(\mathbf{r})$ under the constraint that the box $\Omega$ contains $N_{e}$ electrons. This leads to the following coupled equations:

$$
\begin{gathered}
\frac{\hbar^{2}}{2 m}\left[3 \pi^{2} n_{e}(\mathbf{r})\right]^{2 / 3}+V_{\text {eff }}(\mathbf{r})=\mu, \\
V_{\text {eff }}(\mathbf{r})=V_{\text {Coulomb }}\left[n_{e}(\mathbf{r})\right]+V_{\mathrm{xc}}\left[n_{e}(\mathbf{r})\right],
\end{gathered}
$$

where $\mu$ denotes the chemical potential, playing the role of a Lagrange multiplier. These equations are to be solved for $n_{e}(\mathbf{r})$ self-consistently.

An adopted algorithm to solve the coupled equations (32) and (33) is shown in Fig. 1.
1: procedure HOW TO SET PSEUdO PARTICLE POSITION

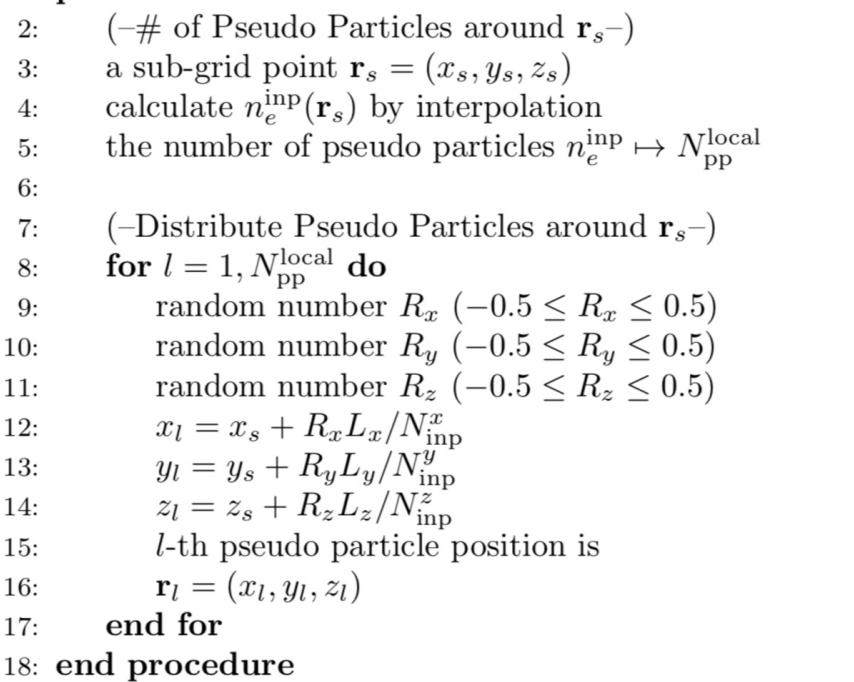

FIG. 2. Algorithm for pseudoparticle distribution.

First, the chemical potential $\mu$ and the electron density $n_{e}^{\text {in }}$ in the real space are guessed so that the total number of electrons within $\Omega$ is $N_{e}$ (line 3). Then, one distributes pseudoparticles according to the guessed $n_{e}^{\text {in }}$ using random numbers (line 7; also see below). The electron density $n_{\mathrm{ps}}$ realized by the pseudoparticle distribution is calculated through Eq. (25) (line 8). The effective potential $V_{\text {eff }}(\mathbf{r})$ is obtained by substituting $n_{\mathrm{ps}}$ into the right-hand side of Eq. (33) (line 9). Then, we update the electron density $n_{e}(\mathbf{r})$ by substituting thus obtained $V_{\text {eff }}(\mathbf{r})$ to Eq. (32) and solving it with respect to $n_{e}$ (line 10), simultaneously updating $\mu$ by a bisection method to satisfy the condition that the total number of electrons is $N_{e}$ (line 11). The updated $n_{e}$ is used as $n_{e}^{\text {in }}$ in the next iteration of the loop (line 13). One repeats the above operations until convergence, $\int_{\Omega} d \mathbf{r}\left|n_{e}^{\text {in }}-n_{e}\right|<\epsilon$, where we set $\epsilon=10^{-7}$ here for crystalline $\mathrm{Al}$ (line 12). After convergence, one distributes the momenta of the pseudoparticles uniformly within the local Fermi radius $p_{f}$ by acceptance-rejection sampling of uniform pseudorandom numbers (lines 17-25).

The algorithm to distribute the pseudoparticles (line 7 in Fig. 1) is shown in Fig. 2.

We introduce subgrid points (line 3 ) by dividing each voxel of the computational grid into $N_{\text {inp }}^{j}$ regions along the $j$ axis $(j=x, y, z)$. The electron density $n_{e}^{\text {inp }}$ on each subgrid point is evaluated, based on the trilinear interpolation from those of the surrounding eight computational grid points, from which one calculates the number of pseudoparticles $N_{\mathrm{pp}}^{\text {local }}$ around the subgrid point (lines 4 and 5). Then, the $N_{\mathrm{pp}}^{\text {local }}$ pseudoparticles are uniformly distributed around the subgrid point using random numbers (lines 8-17).

\section{Linear response}

We evaluate the linear optical response via impulse response by doing dynamical simulations with the initial pseudoparticle momenta $\mathbf{p}_{i}$ shifted from the ground-state values $\mathbf{p}_{i}^{\mathrm{GS}}$ by a small amount $\Delta \mathbf{p}$,

$$
\mathbf{p}_{i}=\mathbf{p}_{i}^{\mathrm{GS}}+\Delta \mathbf{p}
$$


where $\Delta \mathbf{p}=(0,0,0.1$ a.u. $)$ in this study. This is equivalent to the application of an impulse electric field,

$$
\mathbf{E}(t)=-\frac{1}{e} \Delta \mathbf{p} \delta(t),
$$

where $\delta(t)$ is the delta function. Noting that this field has a constant power spectrum across all frequencies, one can readily obtain the optical conductivity as

$$
\sigma_{m n}(\omega)=-\frac{e \hat{J}_{m}(\omega)}{\Delta p_{n}} \quad(m, n=x, y, z),
$$

where $\Delta p_{m}$ and $\hat{J}_{m}(m, n=x, y, z)$ denote the $m$ component of the momentum shift and the temporal Fourier transform of the current density, respectively. The fast Fourier transformation algorithm [61] is used for the evaluation of $\hat{J}_{m}(\omega)$. Assuming isotropic media, the dielectric function $\varepsilon_{m m}(\omega)$, the complex refractive index $n(\omega)$, and the reflectivity $R(\omega)$ are given by

$$
\begin{gathered}
\varepsilon_{m m}(\omega)=1+4 \pi i \frac{\sigma_{m m}(\omega)}{\omega}, \\
n(\omega)=\sqrt{\varepsilon_{m m}(\omega)}, \\
R(\omega)=\left|\frac{\sqrt{\varepsilon_{m m}(\omega)}-1}{\sqrt{\varepsilon_{m m}(\omega)}+1}\right|^{2},
\end{gathered}
$$

respectively, especially, $\varepsilon_{x x}(\omega)=\varepsilon_{y y}(\omega)=\varepsilon_{z z}(\omega)$.

\section{RESULTS}

In this section, we compare the results of the Vlasov-LDA simulations for extended systems described in the previous section with the experimental values as well as the simulation results of the ab initio TDDFT method obtained by the open source code SALMON [36,62-64], which can calculate linear and nonlinear optical properties and the energy transfer from intense laser pulses to materials [65]. We take aluminum as a target material. For Vlasov-LDA, simulation parameters are $N_{s}=10000, N_{e}=12$, and time step $\Delta t=0.025$ a.u. For TDDFT, we employ a norm-conserving pseudopotential [66] and the LDA functional [57], with the number of $k$ points $48^{3}$, number of real-space grids $14^{3}$, and $d t=0.15$ a.u. We assume an external electric field linearly polarized along the $\Gamma-X$ direction of the following temporal profile:

$$
E(t)=E_{0} \sin \left[\omega\left(t-\frac{T}{2}\right)\right] \sin ^{2}\left(\frac{t}{T} \pi\right) \quad(0 \leqslant t \leqslant T),
$$

where $E_{0}$ denotes the field amplitude, $\hbar \omega$ the photon energy, and $T$ the (foot-to-foot) full pulse duration. The corresponding full width at half maximum duration of the laser intensity profile is about $0.36 T$.

\section{A. Linear response}

Let us first discuss the complex optical conductivity, refractive index, extinction coefficient, and reflectivity as a function of photon energy. Despite the simpleness of the Vlasov-LDA approach, its results excellently agree with the TDDFT results (a)

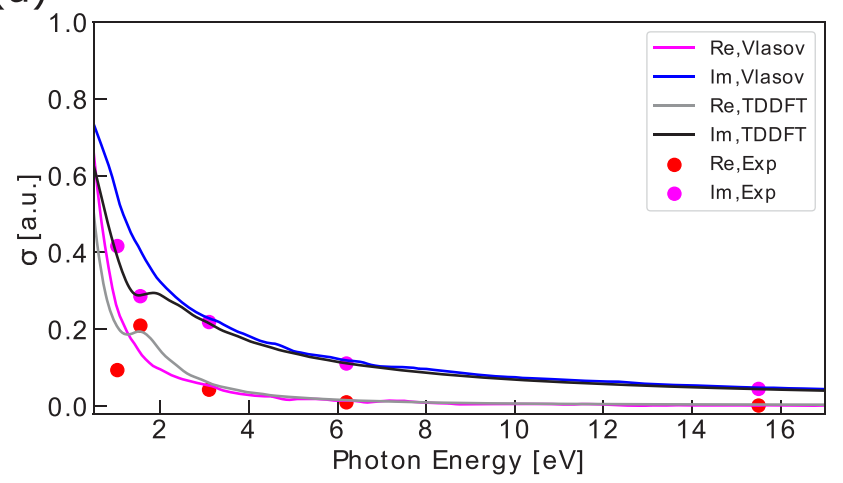

(b)

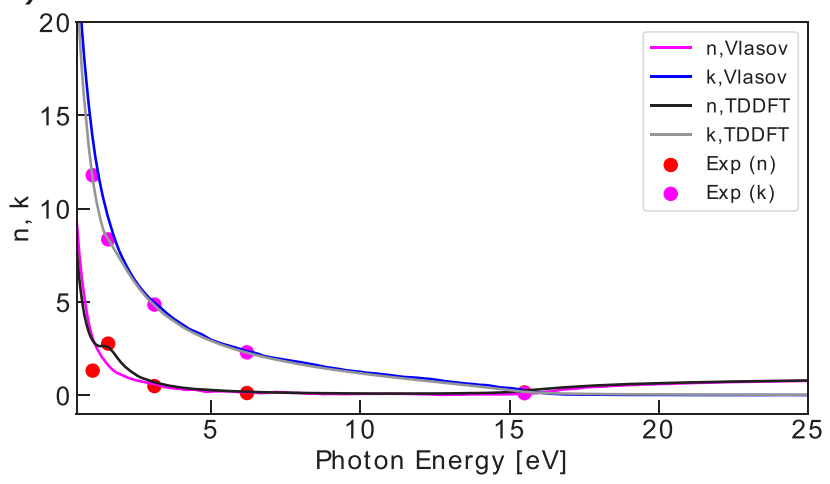

(c)

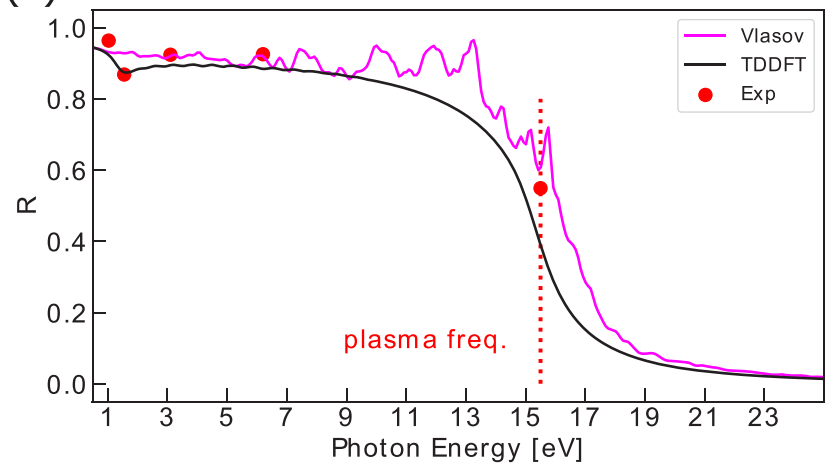

FIG. 3. (a) Optical conductivity $\sigma(\omega)$, (b) refractive index $n$ and extinction coefficient $k$, and (c) reflectivity $R(\omega)$, calculated with the Vlasov-LDA method and TDDFT as well as reported in experimental reference [67]. The experimental values are plotted at $1.03 \mathrm{eV}$ $(1200 \mathrm{~nm}), 1.2 \mathrm{eV}(1030 \mathrm{~nm}), 1.55 \mathrm{eV}(800 \mathrm{~nm}), 3.1 \mathrm{eV}(400 \mathrm{~nm})$, $6.2 \mathrm{eV}(200 \mathrm{~nm})$, and $15.5 \mathrm{eV}(80 \mathrm{~nm})$.

and experimental values (Fig. 3), especially above $2 \mathrm{eV}$ photon energy. The peak and dip around $1.5 \mathrm{eV}$ in the TDDFT results are due to interband absorption, which is not reproduced by the present Vlasov approach, since the latter takes only the single free-electron dispersion into account. Focusing on reflectivity behavior around the plasma frequency, one finds some differences between the two approaches. This difference would be contributions by the above-mentioned interband resonance and nonunity effective mass in TDDFT. We have confirmed it through the decomposition of the response obtained by TDDFT into Drude and Lorentz model components. With the resonance energy set to $1.85 \mathrm{eV}$, the 


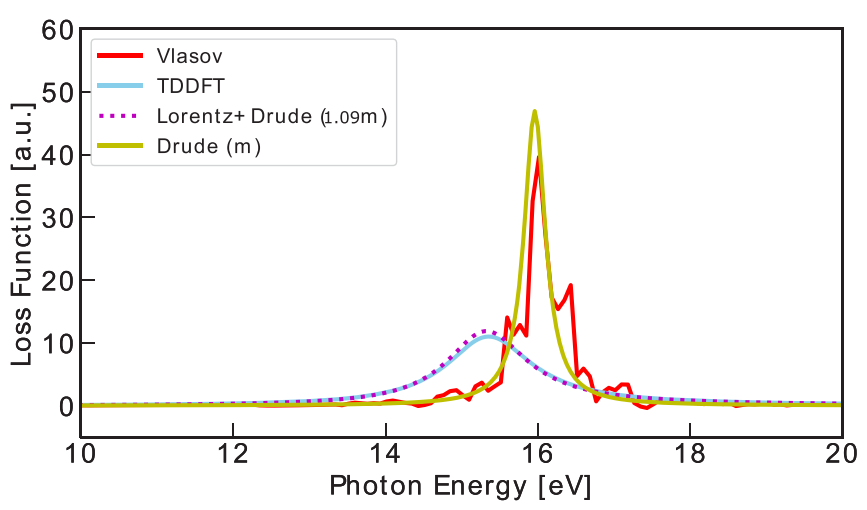

FIG. 4. Loss functions by TDDFT, Vlasov-LDA, combined Drude and Lorentz models $\left(m_{\text {eff }}=1.09 m\right)$, and Drude model $\left(m_{\text {eff }}=\right.$ $m$ ). The peak of the loss function gives plasma frequency.

biggest oscillator around $1.5 \mathrm{eV}$ [68], the estimated effective mass $m_{\text {eff }}$ is $1.09 m$, and the damping constant is $0.51 \mathrm{eV}^{-1}$, consistent with the values reported previously $(1.16 m$ [69] and $0.80 \mathrm{eV}^{-1}$ [68], respectively). The loss functions, $\operatorname{Im} \epsilon(\omega)^{-1}$, are shown in Fig. 4. The TDDFT result is excellently reproduced by the combined Drude and Lorentz contributions with $m_{\text {eff }}=1.09 m$. Although Vlasov-LDA overestimates the plasma frequency compared to TDDFT, it agrees with the Drude model with $m_{\text {eff }}=m$, which implies that the Vlasov dynamics is dominated by free-particle intraband motion.

\section{B. Energy absorption}

Let us next investigate the energy absorption from the laser pulse. We evaluate the energy absorption by the electrons as their energy increment by the pulse irradiation. The energy is calculated as $\Delta E=H_{\mathrm{pp}}(t=\infty)-H_{\mathrm{pp}}(t=0)$ in the VlasovLDA simulation and as $\left\langle h_{\mathrm{KS}}(t=\infty)\right\rangle-\left\langle h_{\mathrm{KS}}(t=0)\right\rangle$ in the TDDFT simulation. We show the fluence dependence for the fixed intensity $\left(10^{12} \mathrm{~W} / \mathrm{cm}^{2}\right)$ at $80 \mathrm{~nm}$ wavelength in Fig. 5 as well as that for the fixed pulse width of $3.8 \mathrm{fs}$ at the ultraviolet (200 and $400 \mathrm{~nm}$ ) wavelengths in Fig. 7 and at the near-infrared wavelengths $(800,1030$, and $1200 \mathrm{~nm})$ in Fig. 8.

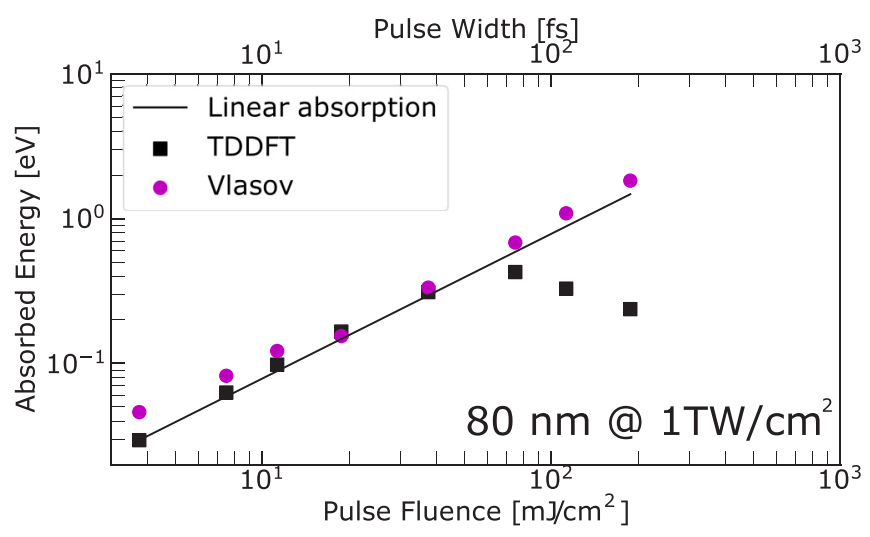

FIG. 5. Calculated absorbed energy vs pulse fluence or pulse width for a fixed intensity $\left(1 \mathrm{TW} / \mathrm{cm}^{2}\right)$. Pink circles: VlasovLDA; black squares: TDDFT; , solid line: linear dependence passing through the square (TDDFT) for the 3.8-fs pulse.

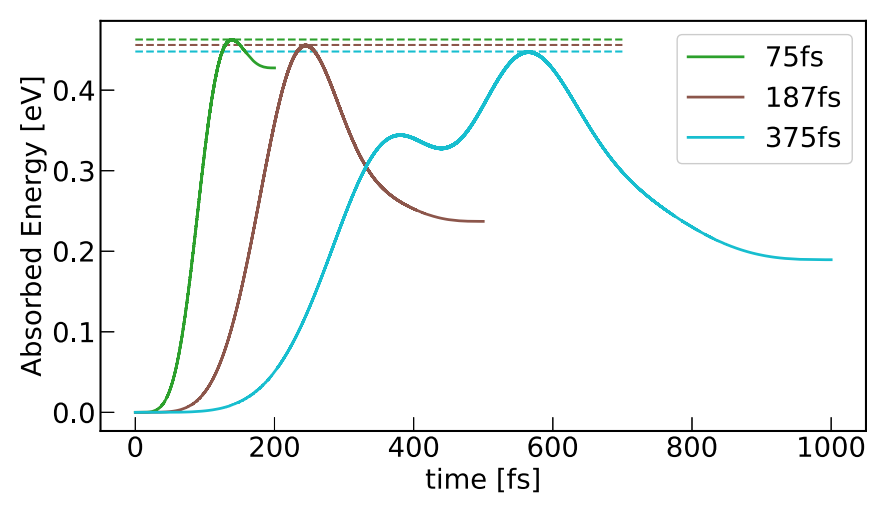

FIG. 6. Solid lines: temporal evolution of the absorbed energy for three different pulse widths 75, 187, and 375 fs. Dashed lines: maximum values for each pulse width.

We see in Fig. 5 that both Vlasov-LDA and TDDFT results are linear in fluence and agree well with each other in the lower fluence region $\left(\lesssim 50 \mathrm{~mJ} / \mathrm{cm}^{2}\right)$. On the other hand, the Vlasov-LDA does not reproduce the TDDFT results for the higher fluence, where the latter deviate from the linear behavior and even decrease with increasing fluence. This difference is due to Rabi-like oscillation [70], as confirmed in Fig. 6, which shows the temporal evolution of absorbed energy for several pulse widths. The maximum electron energy gain
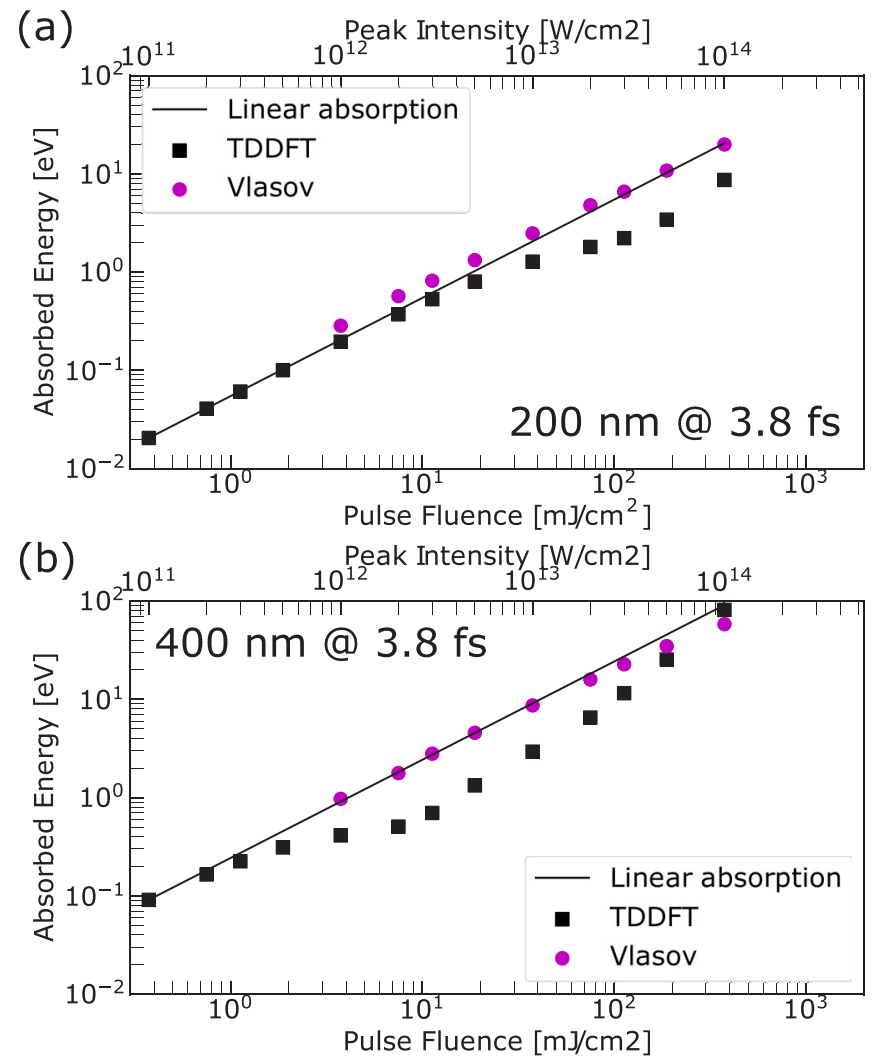

FIG. 7. Absorbed energy vs pulse fluence or peak intensity for a fixed pulse width of $3.8 \mathrm{fs}$ for the case of ultraviolets, (a) $200 \mathrm{~nm}$ and (b) $400 \mathrm{~nm}$ wavelength. Pink circles: Vlasov-LDA; black squares: TDDFT; solid line: linear dependence passing through the square (TDDFT) for $10^{11} \mathrm{~W} / \mathrm{cm}^{2}$ intensity. 

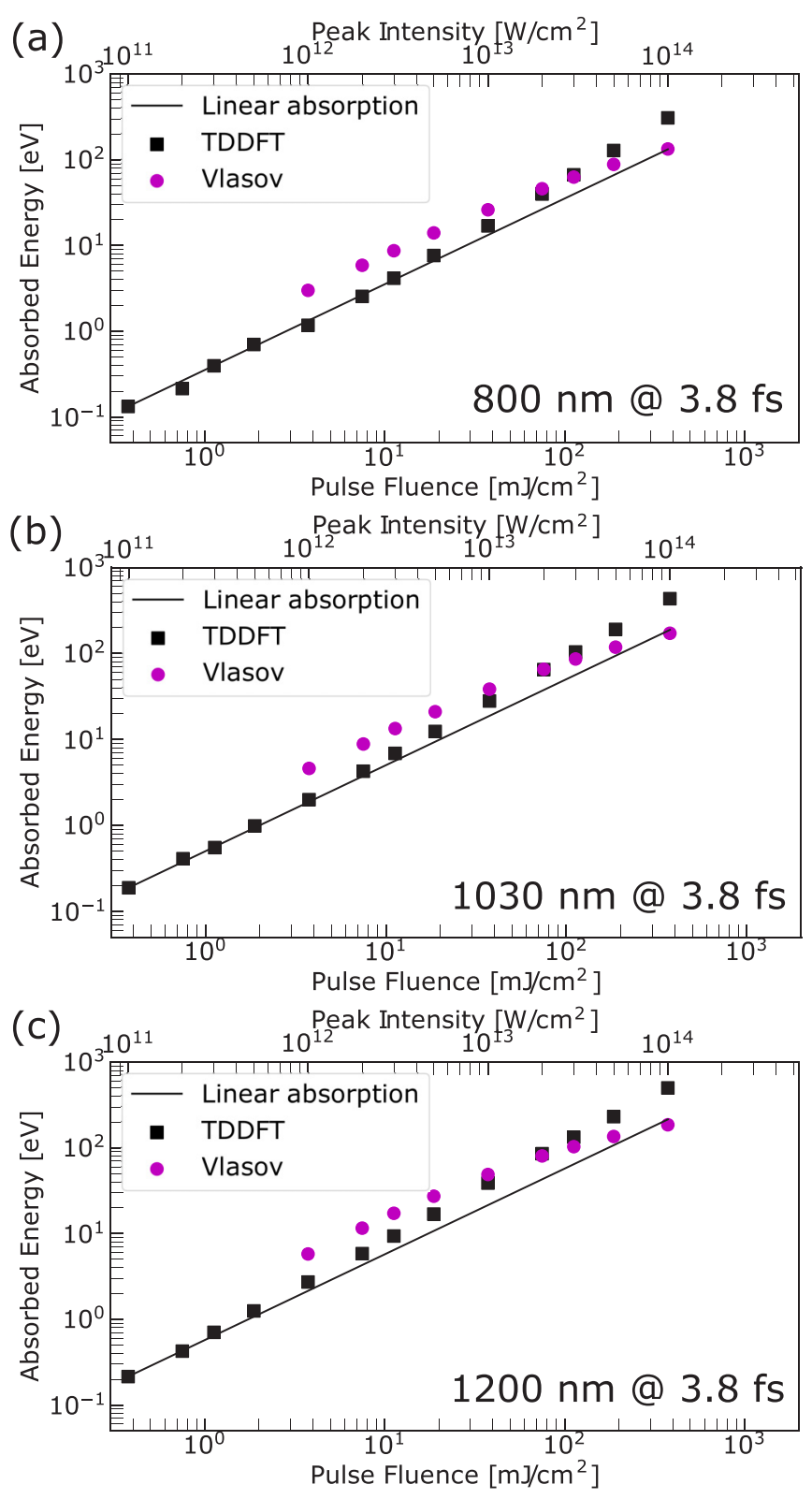

FIG. 8. Absorbed energy vs pulse fluence or peak intensity for a fixed pulse width of $3.8 \mathrm{fs}$ for the case of infrareds, (a) $800 \mathrm{~nm}$, (b) $1030 \mathrm{~nm}$, and (c) $1200 \mathrm{~nm}$ wavelength. Pink circles: VlasovLDA; black squares: TDDFT; solid line: linear dependence passing through the square (TDDFT) for $10^{11} \mathrm{~W} / \mathrm{cm}^{2}$ intensity.

during the pulse, which is indicated by horizontal dashed lines, does not depend much on the pulse width, suggesting Rabi-like coherent oscillation. Thus, there is an optimum pulse width for a fixed intensity in terms of energy absorption. The appearance of an additional bump around $400 \mathrm{fs}$ for the case of 375 fs pulse width might be because the system is not a pure two-level one.

Figures 7 and 8 indicate that the energy absorption calculated by the Vlasov-LDA approach exhibits a linear dependence on fluence or pulse intensity, except for a slight saturation at high intensity. We can see a nonlinear behavior, on the other hand, in the TDDFT results, especially $\gtrsim 40 \mathrm{~mJ} / \mathrm{cm}^{2}$ for $200 \mathrm{~nm}$ and $\gtrsim 2 \mathrm{~mJ} / \mathrm{cm}^{2}$ for $400 \mathrm{~nm}$
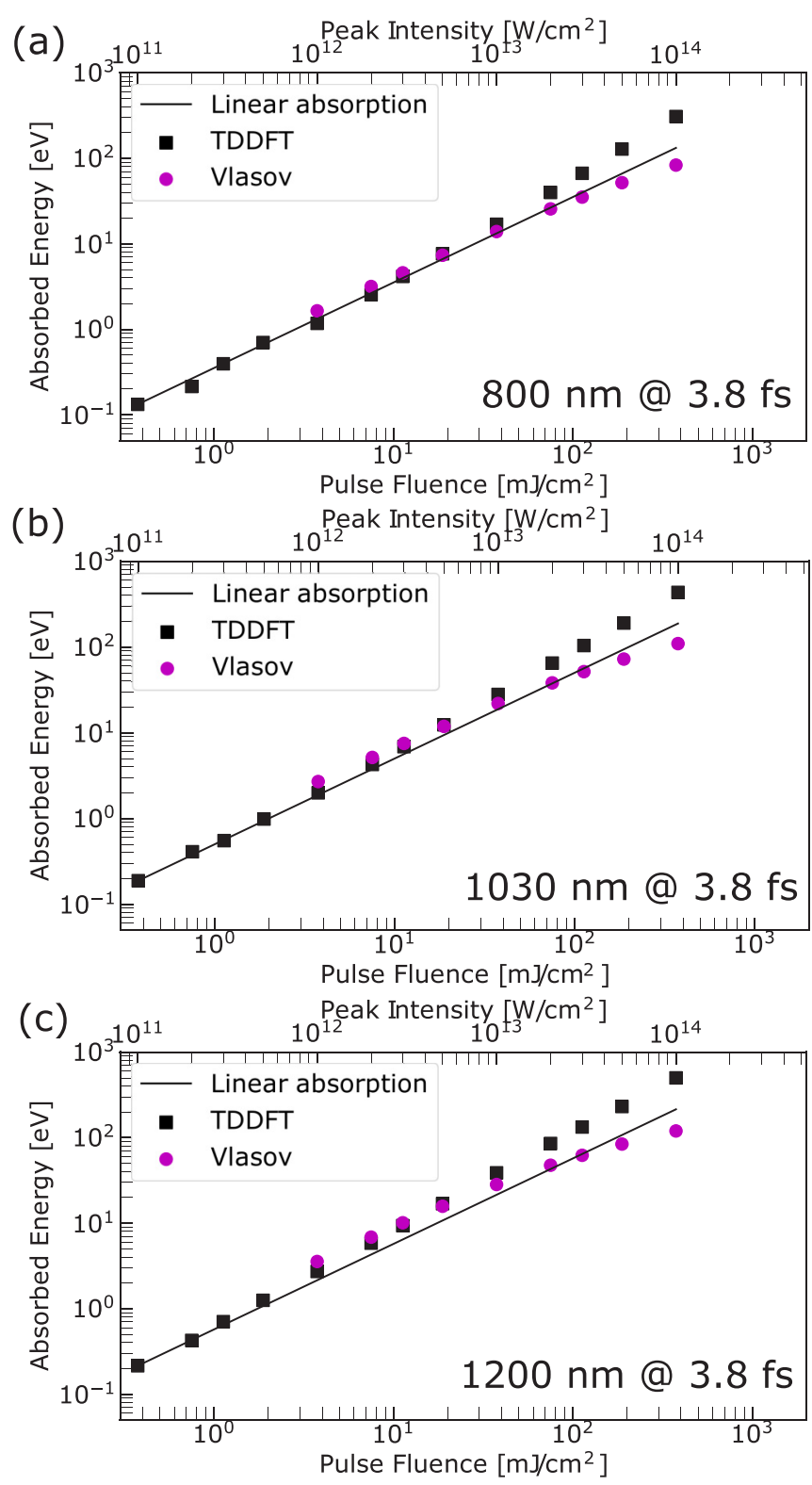

FIG. 9. Absorbed energy vs pulse fluence or peak intensity for a fixed pulse width of $3.8 \mathrm{fs}$ for the case of infrareds, (a) $800 \mathrm{~nm}$, (b) $1030 \mathrm{~nm}$, and (c) $1200 \mathrm{~nm}$. Pink circles: Vlasov-LDA; black squares: TDDFT; solid line: linear dependence passing through the square (TDDFT) for $10^{11} \mathrm{~W} / \mathrm{cm}^{2}$ intensity. $d_{r}$ is set to 1.25 a.u., the result of adjustment referring to $5 \times 10^{12} \mathrm{~W} / \mathrm{cm}^{2}$ at $800 \mathrm{~nm}$.

(Fig. 7). This would be interpreted as saturable absorption as is widely observed in various materials [71]. We could not obtain the Vlasov-LDA results for the low-fluence region $\left(\lesssim 2 \mathrm{~mJ} / \mathrm{cm}^{2}\right)$ because of statistical error. This could be improved by increasing the total number of pseudoparticles, in principle. Nevertheless, the Vlasov-LDA results, if extrapolated to the low-fluence region, appear to agree well with the TDDFT results. For the case of near-infrared excitation (Fig. 8), the Vlasov results overestimate the electron energy absorption compared to the TDDFT ones, probably because the present Vlasov approach cannot reproduce interband transitions around $1.5 \mathrm{eV}$, as has been discussed in Fig. 3 . 
(a)

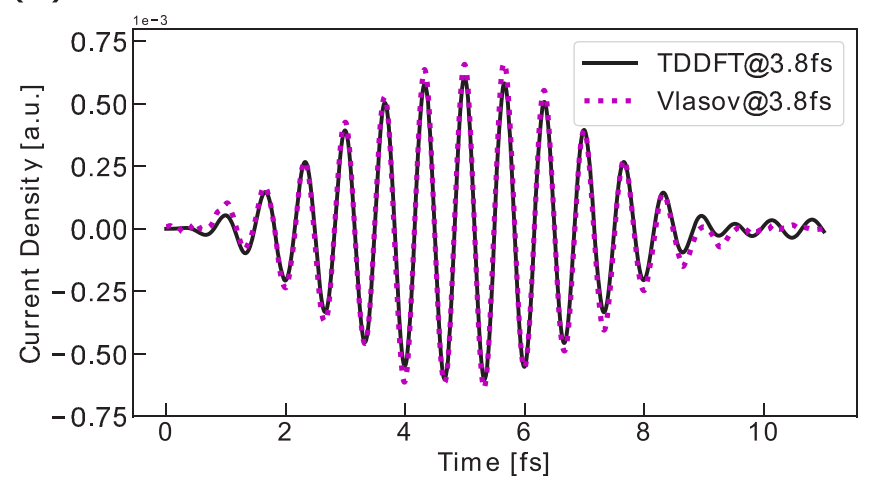

(b)

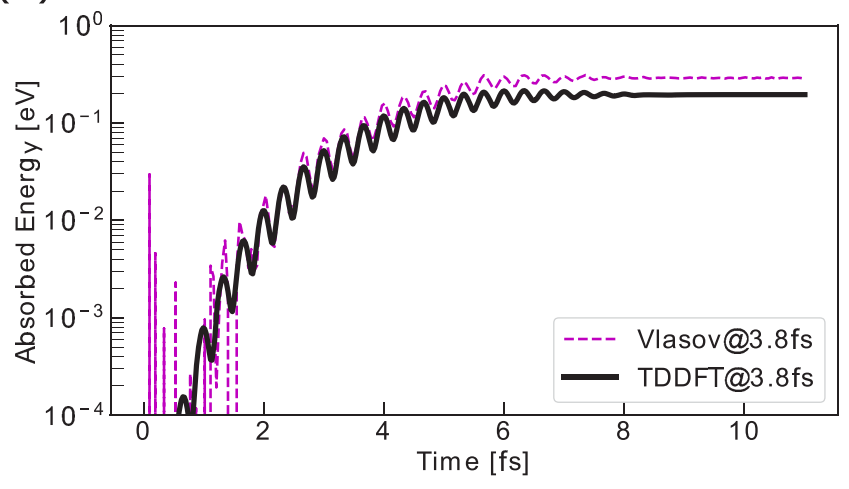

FIG. 10. (a) Time-dependent current density $J(t)$ and (b) electron energy absorption $\Delta E(t)$. Pink dashed lines: Vlasov-LDA; black solid lines: TDDFT.

Nevertheless, the agreement in Fig. 8 is fair. We have adjusted $d_{r}=1.1$ a.u. to achieve overall agreement between the Vlasov and TDDFT results for linear response over a wide range of photon energy $(2-25 \mathrm{eV})$, as mentioned above. If we choose to give priority to agreement in the near-infrared range instead, one can improve it by tuning $d_{r}$ to 1.25 a.u. (Fig. 9). The nonlinear behavior in the TDDFT results appearing at high intensity in Figs. 8 and 9 may suggest the onset of multiphoton excitation.

Figure 10 shows the temporal evolution of the current density and absorbed energy for $10^{12} \mathrm{~W} / \mathrm{cm}^{2}$ peak intensity, $200 \mathrm{~nm}$ wavelength, and $3.8 \mathrm{fs}$ pulse width. Again, overall, the Vlasov results excellently reproduce the TDDFT results. In Fig. 10(b), although energy fluctuation due to the pseudoparticle statistical error is seen at $<2 \mathrm{fs}$, it becomes negligible at the end of the pulse.

Our code is partially parallelized using OpenMP and MPI. One of the most time-consuming parts is the Fourier transformation, which is computed by the naive approach. Nevertheless, the computational time of the present VlasovLDA code is typically only $1 / 20$ of that of TDDFT using the SALMON code. With more sophistication and parallelization, the efficiency of the Vlasov-LDA method will be further improved, which will be advantageous for applications such as parameter optimization in laser material processing.

\section{CONCLUSIONS}

We have extended the Vlasov-LDA semiclassical approach and implemented it with the pseudoparticle method to periodic systems in order to compute the electron dynamics in solids, especially in metals, under ultrashort intense laser pulses. The Vlasov equation can be regarded as the leading order of a semiclassical $\hbar$ expansion of the time-dependent Kohn-Sham equations. The electronic distribution function is expressed by pseudoparticles, incorporating the periodic boundary condition. They play the role of Lagrangian markers embedded randomly in the electron gas. The initial distribution is calculated from the Thomas-Fermi model.

We have applied this approach to crystalline aluminum. Although the method has only one adjustable parameter $d_{r}$, the calculated optical conductivity, refractive index, extinction coefficient, and reflectivity as well as energy absorption are overall in excellent agreement with the TDDFT and experimental results over a wide range of photon energy and fluence, demonstrating the capability of the present approach to accurately describe the dynamics of metallic conduction-band electrons. On the other hand, the Vlasov results deviate from the TDDFT ones around $1.5 \mathrm{eV}$ photon energy, where interband transitions are involved, and at the high-fluence region, where a Rabi-like oscillation takes place.

The next step will be to incorporate electron-electron collisions, as has been done for metal clusters [45]. This extension will offer another advantage over TDDFT, in addition to the reduced computational cost, since the description of electron-electron collisions is limited in TDDFT. Vlasov-LDA is expected to provide valuable insights into complex lasermaterial processing if we further couple it with molecular dynamics [46] and electromagnetic field analysis [64]. The Vlasov approach will also be able to be applied to nonperiodic systems.

\section{ACKNOWLEDGMENTS}

We wish to express our gratitude to Kazuhiro Yabana for private discussions. This research was supported by MEXT Quantum Leap Flagship Program (MEXT Q-LEAP) Grant No. JPMXS0118067246. This research was also partially supported by JSPS KAKENHI Grant No. 20H05670, JST COI Grant No. JPMJCE1313, and JST CREST under Grant No. JPMJCR16N5. M.T. gratefully acknowledges support from the Graduate School of Engineering, The University of Tokyo, Graduate Student Special Incentives Program. M.T. also gratefully acknowledges support through crowd funding platform academist by Misako Abe, Daigo Oue, Miho Otsuka, Yusaku Karibe, Ayano Sakai, Yushi Sakai, Shunsuke A. Sato, Ryosuke Shibato, Hitomi Suto, Tomoharu Sunouchi, Hideo Takahashi, and Yusuke Tokunaga. The numerical calculations are partially performed on supercomputers Oakbridge-CX, sekirei, ohtaka (the University of Tokyo), and SGI ICE X at Japan Atomic Energy Agency (JAEA). This research is partially supported by Initiative on Promotion of Supercomputing for Young or Women Researchers, Information Technology Center, The University of Tokyo. 
[1] S. Ghimire, A. D. DiChiara, E. Sistrunk, P. Agostini, L. F. DiMauro, and D. A. Reis, Observation of high-order harmonic generation in a bulk crystal, Nat. Phys. 7, 138 (2011).

[2] G. Vampa, T. Hammond, N. Thiré, B. Schmidt, F. Légaré, C. McDonald, T. Brabec, and P. Corkum, Linking high harmonics from gases and solids, Nature (London) 522, 462 (2015).

[3] Y. S. You, D. A. Reis, and S. Ghimire, Anisotropic highharmonic generation in bulk crystals, Nat. Phys. 13, 345 (2017).

[4] G. Ndabashimiye, S. Ghimire, M. Wu, D. A. Browne, K. J. Schafer, M. B. Gaarde, and D. A. Reis, Solid-state harmonics beyond the atomic limit, Nature (London) 534, 520 (2016).

[5] Y. Morimoto, Y. Shinohara, M. Tani, B.-H. Chen, K. L. Ishikawa, and P. Baum, Asymmetric single-cycle control of valence electron motion in polar chemical bonds, Optica 8, 382 (2021).

[6] C. Schäfer, H. M. Urbassek, and L. V. Zhigilei, Metal ablation by picosecond laser pulses: A hybrid simulation, Phys. Rev. B 66, 115404 (2002).

[7] C. Kerse, H. Kalaycıoğlu, P. Elahi, B. Çetin, D. K. Kesim, Ö. Akçaalan, S. Yavaş, M. D. Aşık, B. Öktem, H. Hoogland, R. Holzwarth, and F. Ö. Ilday, Ablation-cooled material removal with ultrafast bursts of pulses, Nature (London) 537, 84 (2016).

[8] J.-P. Colombier, P. Combis, F. Bonneau, R. Le Harzic, and E. Audouard, Hydrodynamic simulations of metal ablation by femtosecond laser irradiation, Phys. Rev. B 71, 165406 (2005).

[9] V. Schmidt, W. Husinsky, and G. Betz, Dynamics of Laser Desorption and Ablation of Metals at the Threshold on the Femtosecond Time Scale, Phys. Rev. Lett. 85, 3516 (2000).

[10] K.-H. Leitz, B. Redlingshöfer, Y. Reg, A. Otto, and M. Schmidt, Metal ablation with short and ultrashort laser pulses, Phys. Procedia 12, 230 (2011).

[11] B. N. Chichkov, C. Momma, S. Nolte, F. Von Alvensleben, and A. Tünnermann, Femtosecond, picosecond and nanosecond laser ablation of solids, Appl. Phys. A 63, 109 (1996).

[12] X. Liu, D. Du, and G. Mourou, Laser ablation and micromachining with ultrashort laser pulses, IEEE J. Quantum Electron. 33, 1706 (1997).

[13] S. Amoruso, G. Ausanio, R. Bruzzese, M. Vitiello, and X. Wang, Femtosecond laser pulse irradiation of solid targets as a general route to nanoparticle formation in a vacuum, Phys. Rev. B 71, 033406 (2005).

[14] E. G. Gamaly, The physics of ultra-short laser interaction with solids at non-relativistic intensities, Phys. Rep. 508, 91 (2011).

[15] N. Medvedev, H. O. Jeschke, and B. Ziaja, Nonthermal phase transitions in semiconductors induced by a femtosecond extreme ultraviolet laser pulse, New J. Phys. 15, 015016 (2013).

[16] R. R. Gattass and E. Mazur, Femtosecond laser micromachining in transparent materials, Nat. Photonics 2, 219 (2008).

[17] I. Mirza, N. M. Bulgakova, J. Tomáštík, V. Michálek, O. Haderka, L. Fekete, and T. Mocek, Ultrashort pulse laser ablation of dielectrics: Thresholds, mechanisms, role of breakdown, Sci. Rep. 6, 39133 (2016).

[18] E. Silaeva, A. Vella, N. Sevelin-Radiguet, G. Martel, B. Deconihout, and T. Itina, Ultrafast laser-triggered field ion emission from semiconductor tips, New J. Phys. 14, 113026 (2012).

[19] B. Rethfeld, D. S. Ivanov, M. E. Garcia, and S. I. Anisimov, Modelling ultrafast laser ablation, J. Phys. D 50, 193001 (2017).
[20] B. Rethfeld, Unified Model for the Free-Electron Avalanche in Laser-Irradiated Dielectrics, Phys. Rev. Lett. 92, 187401 (2004).

[21] A. Rudenko, J.-P. Colombier, and T. E. Itina, From random inhomogeneities to periodic nanostructures induced in bulk silica by ultrashort laser, Phys. Rev. B 93, 075427 (2016).

[22] B. Chimier, O. Utéza, N. Sanner, M. Sentis, T. Itina, P. Lassonde, F. Légaré, F. Vidal, and J. C. Kieffer, Damage and ablation thresholds of fused-silica in femtosecond regime, Phys. Rev. B 84, 094104 (2011).

[23] P. Lorazo, L. J. Lewis, and M. Meunier, Thermodynamic pathways to melting, ablation, and solidification in absorbing solids under pulsed laser irradiation, Phys. Rev. B 73, 134108 (2006).

[24] J. Thorstensen and S. Erik Foss, Temperature dependent ablation threshold in silicon using ultrashort laser pulses, J. Appl. Phys. 112, 103514 (2012).

[25] T. Kondo, S. Matsuo, S. Juodkazis, V. Mizeikis, and H. Misawa, Multiphoton fabrication of periodic structures by multibeam interference of femtosecond pulses, Appl. Phys. Lett. 82, 2758 (2003).

[26] A. K. Upadhyay, N. A. Inogamov, B. Rethfeld, and H. M. Urbassek, Ablation by ultrashort laser pulses: Atomistic and thermodynamic analysis of the processes at the ablation threshold, Phys. Rev. B 78, 045437 (2008).

[27] D. Ivanov, A. Kuznetsov, V. Lipp, B. Rethfeld, B. Chichkov, M. Garcia, and W. Schulz, Short laser pulse nanostructuring of metals: Direct comparison of molecular dynamics modeling and experiment, Appl. Phys. A 111, 675 (2013).

[28] D. S. Ivanov, V. P. Lipp, A. Blumenstein, F. Kleinwort, V. P. Veiko, E. Yakovlev, V. Roddatis, M. E. Garcia, B. Rethfeld, J. Ihlemann, and P. Simon, Experimental and Theoretical Investigation of Periodic Nanostructuring of Au with Ultrashort UV Laser Pulses Near the Damage Threshold, Phys. Rev. Appl. 4, 064006 (2015).

[29] T. E. Itina, K. Gouriet, L. V. Zhigilei, S. Noël, J. Hermann, and M. Sentis, Mechanisms of small clusters production by short and ultra-short laser ablation, Appl. Surf. Sci. 253, 7656 (2007).

[30] B. J. Garrison, T. E. Itina, and L. V. Zhigilei, Limit of overheating and the threshold behavior in laser ablation, Phys. Rev. E 68, 041501 (2003).

[31] S. Sakabe, M. Hashida, S. Tokita, S. Namba, and K. Okamuro, Mechanism for self-formation of periodic grating structures on a metal surface by a femtosecond laser pulse, Phys. Rev. B 79, 033409 (2009).

[32] M. Ishino, N. A. Inogamov, S. Tamotsu, V. V. Zhakhovsky, N. Hasegawa, I. Y. Skobelev, A. Y. Faenov, T. A. Pikuz, K. Mikami, T. Kawachi, and M. Nishikino, Study of damage structure formation on aluminum film targets by picosecond soft $\mathrm{x}$-ray laser ablation around threshold region, Appl. Phys. A 124, 649 (2018).

[33] J. P. Anthes, M. A. Gusinow, and M. K. Matzen, Experimental Observation and Numerical Simulations of Laser-Driven Ablation, Phys. Rev. Lett. 41, 1300 (1978).

[34] B. J. Simonds, J. Sowards, J. Hadler, E. Pfeif, B. Wilthan, J. Tanner, C. Harris, P. Williams, and J. Lehman, TimeResolved Absorptance and Melt Pool Dynamics During Intense Laser Irradiation of a Metal, Phys. Rev. Appl. 10, 044061 (2018). 
[35] C. Wu and L. V. Zhigilei, Microscopic mechanisms of laser spallation and ablation of metal targets from largescale molecular dynamics simulations, Appl. Phys. A 114, 11 (2014).

[36] M. Noda, S. A. Sato, Y. Hirokawa, M. Uemoto, T. Takeuchi, S. Yamada, A. Yamada, Y. Shinohara, M. Yamaguchi, K. Iida, I. Floss, T. Otobe, K.-M. Lee, K. Ishimura, T. Boku, G. F. Bertsch, K. Nobusada, and K. Yabana, SALMON: Scalable ab-initio light-matter simulator for optics and nanoscience, Comput. Phys. Commun. 235, 356 (2019).

[37] K. Yabana, T. Sugiyama, Y. Shinohara, T. Otobe, and G. F. Bertsch, Time-dependent density functional theory for strong electromagnetic fields in crystalline solids, Phys. Rev. B 85, 045134 (2012).

[38] A. D. Baczewski, L. Shulenburger, M. P. Desjarlais, S. B. Hansen, and R. J. Magyar, X-ray Thomson Scattering in Warm Dense Matter without the Chihara Decomposition, Phys. Rev. Lett. 116, 115004 (2016).

[39] T. Otobe, Wavelength Dependence of the Laser-Excitation Process on a Silicon Surface, Phys. Rev. Appl. 13, 024062 (2020).

[40] K. Kaneshima, Y. Shinohara, K. Takeuchi, N. Ishii, K. Imasaka, T. Kaji, S. Ashihara, K. L. Ishikawa, and J. Itatani, PolarizationResolved Study of High Harmonics from Bulk Semiconductors, Phys. Rev. Lett. 120, 243903 (2018).

[41] H. Hirori, P. Xia, Y. Shinohara, T. Otobe, Y. Sanari, H. Tahara, N. Ishii, J. Itatani, K. L. Ishikawa, T. Aharen, M. Ozaki, A. Wakamiya, and Y. Kanemitsu, High-order harmonic generation from hybrid organic-inorganic perovskite thin films, APL Mater. 7, 041107 (2019).

[42] Y. Sanari, H. Hirori, T. Aharen, H. Tahara, Y. Shinohara, K. L. Ishikawa, T. Otobe, P. Xia, N. Ishii, J. Itatani, S. A. Sato, and Y. Kanemitsu, Role of virtual band population for high harmonic generation in solids, Phys. Rev. B 102, 041125(R) (2020).

[43] K. Ishikawa and T. Blenski, Particle-in-cell simulations of multiple ionization of small molecules in a strong laser field, Phys. Rev. A 61, 063408 (2000).

[44] D. Palade and V. Baran, Optical response of $\mathrm{C}_{60}$ fullerene from a time dependent Thomas Fermi approach, J. Phys. B: At., Mol. Opt. Phys. 48, 185102 (2015).

[45] E. Giglio, E. Suraud, and P.-G. Reinhard, Semi-classical description of ionic and electronic dynamics in metal clusters, Ann. Phys. 11, 291 (2002).

[46] T. Fennel, G. Bertsch, and K.-H. Meiwes-Broer, Ionization dynamics of simple metal clusters in intense fields by the Thomas-Fermi-Vlasov method, Eur. Phys. J. D 29, 367 (2004).

[47] J. Köhn, R. Redmer, K.-H. Meiwes-Broer, and T. Fennel, Nonresonant absorption enhancement in laser-excited simple metal clusters through electron-electron collisions, Phys. Rev. A 77, 033202 (2008).

[48] L. Plagne, J. Daligault, K. Yabana, T. Tazawa, Y. Abe, and C. Guet, Semiclassical versus quantal time-dependent meanfield descriptions of electron dynamics in ion-cluster collisions, Phys. Rev. A 61, 033201 (2000).

[49] A. Domps, P.-G. Reinhard, and E. Suraud, Fermionic Vlasov propagation for Coulomb interacting systems, Ann. Phys. 260, 171 (1997).
[50] A. Domps, P. L'Eplattenier, P. Reinhard, and E. Suraud, The Vlasov equation for Coulomb systems and the Husimi picture, Ann. Phys. 509, 455 (1997).

[51] F. Calvayrac, P. Reinhard, and E. Suraud, Ionic structure and plasmon response in sodium clusters, J. Phys. B: At., Mol. Opt. Phys. 31, 1367 (1998).

[52] L. Féret, E. Suraud, F. Calvayrac, and P. Reinhard, On the electron dynamics in metal clusters: A Vlasov approach, J. Phys. B: At., Mol. Opt. Phys. 29, 4477 (1996).

[53] Y. Abe, P. Bozek, and K. Yabana, Time-dependent local density approximation for cluster-ion collisions, Czech. J. Phys. 48, 838 (1998).

[54] E. Runge and E. K. U. Gross, Density-Functional Theory for Time-Dependent Systems, Phys. Rev. Lett. 52, 997 (1984).

[55] E. P. Wigner, On the quantum correction for thermodynamic equilibrium, Phys. Rev. 40, 749 (1932).

[56] C. Fiolhais, J. P. Perdew, S. Q. Armster, J. M. MacLaren, and M. Brajczewska, Dominant density parameters and local pseudopotentials for simple metals, Phys. Rev. B 51, 14001 (1995).

[57] J. P. Perdew and A. Zunger, Self-interaction correction to density-functional approximations for many-electron systems, Phys. Rev. B 23, 5048 (1981).

[58] S. Tanaka, K. Yoshikawa, T. Minoshima, and N. Yoshida, Multidimensional Vlasov-Poisson simulations with high-order monotonicity- and positivity-preserving schemes, Astrophys. J. 849, 76 (2017).

[59] G. F. Bertsch and S. D. Gupta, A guide to microscopic models for intermediate energy heavy ion collisions, Phys. Rep. 160 189 (1988).

[60] L. Verlet, Computer "experiments" on classical fluids. I. thermodynamical properties of Lennard-Jones molecules, Phys. Rev. 159, 98 (1967).

[61] J. W. Cooley and J. W. Tukey, An algorithm for the machine calculation of complex Fourier series, Math. Comput. 19, 297 (1965).

[62] G. F. Bertsch, J.-I. Iwata, A. Rubio, and K. Yabana, Real-space, real-time method for the dielectric function, Phys. Rev. B 62, 7998 (2000).

[63] K. Yabana and G. F. Bertsch, Time-dependent localdensity approximation in real time, Phys. Rev. B 54, 4484 (1996).

[64] S. A. Sato and K. Yabana, Maxwell + TDDFT multi-scale simulation for laser-matter interaction, J. Adv. Simul. Sci. Eng. 1, 98 (2014).

[65] K.-M. Lee, C. Min Kim, S. A. Sato, T. Otobe, Y. Shinohara, K. Yabana, and T. Moon Jeong, First-principles simulation of the optical response of bulk and thin-film $\alpha$-quartz irradiated with an ultrashort intense laser pulse, J. Appl. Phys. 115, 053519 (2014).

[66] M. Fuchs and M. Scheffler, Ab initio pseudopotentials for electronic structure calculations of poly-atomic systems using density-functional theory, Comput. Phys. Commun. 119, 67 (1999).

[67] A. D. Rakić, Algorithm for the determination of intrinsic optical constants of metal films: Application to aluminum, Appl. Opt 34, 4755 (1995).

[68] T. Gharbi, D. Barchiesi, S. Kessentini, and R. Maâlej, Fitting optical properties of metals by Drude-Lorentz and 
partial-fraction models in the $[0.5 ; 6] \mathrm{eV}$ range, Opt. Mater. Express 10, 1129 (2020).

[69] A. I. Kiselev, L. A. Akashev, and V. I. Kononenko, Effective electron mass in melts of aluminum, cerium, and Al-3 at.\% Ce binary system, Tech. Phys. 49, 302 (2004).
[70] A. Yamada and K. Yabana, Energy transfer from intense laser pulse to dielectrics in time-dependent density functional theory, Eur. Phys. J. D 73, 87 (2019).

[71] M. Uemoto, S. Kurata, N. Kawaguchi, and K. Yabana, Firstprinciples study of ultrafast and nonlinear optical properties of graphite thin films, Phys. Rev. B 103, 085433 (2021). 